\title{
Media Optimization, Strain Compatibility, and Low-Shear Modeled Microgravity Exposure of Synthetic Microbial Communities for Urine Nitrification in Regenerative Life-Support Systems
}

\author{
Chiara Ilgrande, ${ }^{1}$ Tom Defoirdt, ${ }^{1}$ Siegfried E. Vlaeminck, ${ }^{1,2}$ Nico Boon, ${ }^{1}$ and Peter Clauwaert ${ }^{1}$
}

\begin{abstract}
Urine is a major waste product of human metabolism and contains essential macro- and micronutrients to produce edible microorganisms and crops. Its biological conversion into a stable form can be obtained through urea hydrolysis, subsequent nitrification, and organics removal, to recover a nitrate-enriched stream, free of oxygen demand. In this study, the utilization of a microbial community for urine nitrification was optimized with the focus for space application.

To assess the role of selected parameters that can impact ureolysis in urine, the activity of six ureolytic heterotrophs (Acidovorax delafieldii, Comamonas testosteroni, Cupriavidus necator, Delftia acidovorans, Pseudomonas fluorescens, and Vibrio campbellii) was tested at different salinities, urea, and amino acid concentrations. The interaction of the ureolytic heterotrophs with a nitrifying consortium (Nitrosomonas europaea ATCC 19718 and Nitrobacter winogradskyi ATCC 25931) was also tested. Lastly, microgravity was simulated in a clinostat utilizing hardware for in-flight experiments with active microbial cultures. The results indicate salt inhibition of the ureolysis at $30 \mathrm{mS} \mathrm{cm}$, while amino acid nitrogen inhibits ureolysis in a straindependent manner. The combination of the nitrifiers with $C$. necator and $V$. campbellii resulted in a complete halt of the urea hydrolysis process, while in the case of A. delafieldii incomplete nitrification was observed, and nitrite was not oxidized further to nitrate. Nitrate production was confirmed in all the other communities; however, the other heterotrophic strains most likely induced oxygen competition in the test setup, and nitrite accumulation was observed. Samples exposed to low-shear modeled microgravity through clinorotation behaved similarly to the static controls. Overall, nitrate production from urea was successfully demonstrated with synthetic microbial communities under terrestrial and simulated space gravity conditions, corroborating the application of this process in space. Key Words: Nitrosomonas europaea-Nitrogen recovery-Nitrobacter winogradskyi-Heterotrophs-MELiSSA. Astrobiology 19, 1353-1362.
\end{abstract}

\section{Introduction}

$\mathbf{N}$ ITROGEN IS A KEY element to produce nutritional vegetables or edible microorganisms, and its recovery from organic wastes is pivotal for regenerative life-support system technologies. The largest nitrogen flow in a regenerative lifesupport system is human urine. Assuming a dietary protein intake of $0.8-1.5 \mathrm{~g}$ protein $\mathrm{kg}^{-1}$ for a crew member with a body weight of $65-85 \mathrm{~kg}$, nitrogen secretion through urine can be estimated between 7 and $16 \mathrm{~g} \mathrm{~N} \mathrm{~d}^{-1}$, mainly ( $\sim 90 \%$ ) in the form of urea (Clauwaert et al., 2017).
Although urea can be directly assimilated by plants (Witte, 2011), normally it is rapidly hydrolyzed by microbial urease it into ammonia and carbamate, which then spontaneously decays into $\mathrm{CO}_{2}$ and an additional ammonia molecule. Plants can also take up inorganic nitrogen forms, such as ammonia and nitrate (Andrews et al., 2013). Of the two, ammonia poses a hazard in regenerative life-support systems due to its volatile state, as it can accumulate to toxic levels in the atmosphere. Ammonia inhalation has been linked to reduced pulmonary functionality and severe lung symptoms, including asthma, coughing, burns, and irritation

\footnotetext{
${ }^{1}$ Center for Microbial Ecology and Technology, Ghent University, Gent, Belgium.

${ }^{2}$ Research Group of Sustainable Energy, Air and Water Technology, Department of Bioscience Engineering, University of Antwerp, Antwerpen, Belgium.
} 
of the respiratory tract (EPA, 2016). Additionally, ammonia buildup can produce phytotoxic effect, affecting plant development (Clauwaert et al., 2017). Hence, its stabilization into a safer nitrogen species is recommended.

Nitrification is the process used to convert ammonia into the less toxic and nonvolatile nitrate. This conversion can be biologically performed by either a combination of ammoniaoxidizing bacteria (AOB) or ammonia-oxidizing archaea (AOA) and nitrite-oxidizing bacteria (NOB) (Jetten, 2008) or by complete ammonia-oxidizing bacteria (comammox) (Daims et al., 2015; van Kessel et al., 2015). Nitrification with AOB and NOB has been specifically studied for application in regenerative life-support systems, and a nitrification compartment with Nitrosomonas europaea as AOB and Nitrobacter winogradskyi as NOB has been developed and validated for application in the Micro-Ecological Life Support System Alternative (MELiSSA), the European Space Agency's regenerative life-support system (Pérez et al., 2004; Montràs et al., 2008; Cruvellier et al., 2016). While a combination of ureolysis and nitrification has been repeatedly demonstrated with open communities (e.g., De Paepe et al., 2018; Muys et al., 2018), this has not yet been reported with reductionistic synthetic communities. Since ammonia oxidation is commonly considered the limiting step of nitrification, so far little focus has been reserved to the complete urea hydrolysis and subsequent nitrification process, with only one synthetic community being described (Lindeboom et al., 2018). To include urea hydrolysis and nitrification as bioprocesses in the MELiSSA nutrient loop, the nitrifying compartment of MELiSSA needs to be supplemented with appropriate nonpathogenic ureolytic bacteria, establishing a urea hydrolytic and nitrifying community (Hendrickx and Mergeay, 2007). To this end, in this study key challenges for urine nitrification were identified and tested utilizing different ureolytic strains, namely Acidovorax delafieldii DSMZ 64, Comamonas testosteroni I2 (Boon et al., 2000), Cupriavidus necator DSMZ 13513, Delftia acidovorans DSMZ 14801, Pseudomonas fluorescens DSMZ 50090, and Vibrio campbellii LMG 22895.

Firstly, the impact of dilution on urea hydrolysis was addressed. Urine is a matrix with a variable composition, determined by interpersonal variability, diet, and water intake (Siener and Hesse, 2002; Nagaraj and Mann, 2011). Furthermore, some of its components may interfere with the expression of nonconstitutive ureases. Urea has been reported as an inducer of ureolytic activity in some bacteria, while alternative nitrogen sources have been reported as inhibitors (Mobley et al. 1995; Defoirdt et al., 2017).

To elucidate the impact of salinity and the minimum level of urea to induce ureolysis, the ureolytic activity of the strains was evaluated in an artificial urine salt matrix with different levels of salinity, corresponding to electrical conductivity (EC) levels reported for nitrification systems treating $10 \%$, $20 \%$, and $60 \%$ real urine $\left(5,10\right.$, and $30 \mathrm{mS} \mathrm{cm}{ }^{-1}$, respectively) (Coppens et al., 2016; De Paepe et al., 2018) and a low urea concentration $\left(10,25\right.$, or $50 \mathrm{mg}-\mathrm{N} \mathrm{L}^{-1}$, for each salinity tested). Since urine contains also multiple forms of organic nitrogen (Bouatra et al., 2013), the ureolysis was evaluated in the presence of different concentrations of a mixture of amino acids (130, 65, and $33 \mathrm{mg} \mathrm{L}^{-1}$ ) (Kim et al., 2013).

Although engineering microbial communities and populations allows the development of bioprocesses with func- tionalities that are difficult or impossible for a single species, the challenges represented by detrimental microbial interactions need to be addressed (Brenner et al., 2008). Communities composed of Nitrosomonas europaea and Nitrobacter winogradskyi can efficiently convert ammonia into nitrate for prolonged periods of time (Pérez et al., 2004). However, the introduction of a ureolytic heterotroph might alter or disrupt the process. Synthetic microbial communities combining the ureolytic heterotrophs selected with the AOB N. europaea ATCC 19718 and the NOB $N$. winogradskyi ATCC 25931, which are included in the nitrifying compartment of MELiSSA (Hendrickx et al., 2006), were assembled. The conversion of urea into nitrate was monitored over time, to assess the functionality of the microbial communities.

Finally, regenerative life-support systems and urine nitrification systems will have to be applied in space. However, space conditions can affect the performance of the bioprocess. Bacteria are exposed to a higher radiation dose than on Earth, which is damaging to the cells, and a reduced gravity that alters the fluid dynamics (Benoit and Klaus, 2007). Historically, the effect of microgravity has been controversial. Early reports suggested that growth in microgravity increased bacterial growth rates over cultures grown in normal gravity, while over time this observation was found not to be universally true. Instead, growth rates were dependent on growth media, motility, and the bacterial species and strain, with a recent report suggesting that the motility of the bacteria is the primary cause of this discrepancy (Benoit and Klaus, 2007). While a synthetic community of N. europaea, N. winogradskyi, and Cupriavidus pinatubonensis and the respective pure cultures have recently been shown to withstand storage on an unmanned spaceflight in low Earth orbit for 44 days (Lindeboom et al., 2018), the effect on active cultures is unmapped. In this study, reduced gravity was simulated through clinorotation, with hardware typically utilized for in-flight experiments with active cultures. The bacteria were exposed to low-shear modeled microgravity, a state of constant fluid suspension, such that hydrodynamic forces counterbalance the gravityinduced sedimentation of the bacteria, through clinorotation (Nickerson et al., 2004).

The impact of this condition was evaluated by monitoring the conversion of urea into nitrate.

\section{Materials and Methods}

\subsection{Culture conditions and synthetic communities' assembly}

The ureolytic heterotrophs Acidovorax delafieldii DSMZ 64, Comamonas testosteroni I2, Cupriavidus necator DSMZ 13513, Delftia acidovorans DSMZ 14801, Pseudomonas fluorescens DSMZ 50090, and Vibrio campbellii LMG 22895 were grown in $\mathrm{LB}$ broth at $28^{\circ} \mathrm{C}$ in an orbital shaker (KA, KS 4000 i control, Germany).

The AOB N. europaea ATCC 19718 was grown axenically in ATCC medium 2265 while the NOB N. winogradskyi ATCC 25931 was grown axenically in ATCC medium 480 in $10 \mathrm{~mL}$ aliquots in ventilated $25 \mathrm{~mL}$ cell culture flasks at $28^{\circ} \mathrm{C}$ in the dark with in an orbital shaker (KA, KS 4000 i control, Germany) with gentle shaking. After 2 weeks, new subculture $10 \%(\mathrm{v} / \mathrm{v})$ of the grown cultures was transferred to $9 \mathrm{~mL}$ 
fresh medium. In the case of $N$. winogradskyi, $100 \mathrm{mg} \mathrm{L}^{-1}$ $\mathrm{NO}_{2}{ }^{-} \mathrm{N}$ was added when nitrite was depleted. To adapt the cultures to the urine matrix, a stepwise strategy was applied. At each transfer of the cultures, the standard growth media were mixed with increasing levels of urine salts solution (Table 2) diluted 1:3 with phosphate buffer $(0.5 \mathrm{M}, \mathrm{pH} 7)$, to a final concentration of $33 \%$ urine salts after the first subculture, $67 \%$ after the second, and $100 \%$ after the third. Respectively, $500 \mathrm{mg} \mathrm{NH}_{4}{ }^{+}-\mathrm{N} \mathrm{L}^{-1}$ or $\mathrm{mg} \mathrm{NO}_{2}^{-}-\mathrm{N} \mathrm{L}^{-1}$ was added as substrate to $N$. europaea and $N$. winogradskyi cultures. Cell densities were determined spectrophotometrically at $\mathrm{OD}_{600}$.

The synthetic communities were assembled starting from independently grown cultures of heterotroph and $N$. europaea and $N$. winogradskyi after the adaptation process (Table 1). Each culture was diluted to the same $\mathrm{OD}_{600}$. The complete synthetic microbial community was assembled mixing equal volumes of heterotroph, $N$. europaea, and $N$. winogradskyi to a final $\mathrm{OD}_{600}$ of 0.1 . The nitrifying control without ureolytic strain was assembled by mixing equal volumes of substrate depleted urine salts solution diluted $1: 3$ with phosphate buffer $(0.5 \mathrm{M}, \mathrm{pH} 7), N$. europaea, and $N$. winogradskyi to a final $\mathrm{OD}_{600}$ of 0.066 . Hence, the complete community and the control have the same AOB and NOB amount.

\subsection{Analytical determination of the nitrogen species}

All nitrogen species were determined colorimetrically. The metabolite measurement was performed according to the reaction reported by Yatzidis et al. (1964) for urea, to the Berthelot reaction for ammonia (Bucur et al., 2006), to the Montgomery reaction for nitrite (Montgomery and Dymock, 1961) while nitrate was measured as described by Cataldo et al. (1975). All spectrophotometric measurements were performed with a Microplate Readers Infinite F50 (Tecan group Ltd., Germany).

\subsection{Activity tests}

2.3.1. Effect of urea concentration and salinity. All the tests were performed in triplicates for each strain, in flatbottom 96 multiwell plates incubated at $28^{\circ} \mathrm{C}$ in the dark in a Thermoshaker (MB100-4A, Thermoshakers Aosheng, China). An orbital shaking of $600 \mathrm{rpm}$ was applied to pre-

Table 1. Composition of the Synthetic Communities Used in This STUdy

\begin{tabular}{ll}
\hline Community $n^{\circ}$ & Composition \\
\hline 1 & N. europaea, $N$. winogradskyi \\
2 & N. europaea, $N$. winogradskyi, \\
& A. delafieldii \\
3 & N. europaea, $N$. winogradskyi, \\
C. testosteroni \\
4 & N. europaea, $N$. winogradskyi, \\
& C. necator \\
5 & N. europaea, $N$. winogradskyi, \\
& D. acidovorans \\
6 & N. europaea, $N$. winogradskyi, \\
7 & P. fluorescens \\
7 & N. europaea, $N$. winogradskyi, \\
& V. campbellii \\
\hline
\end{tabular}

Table 2. Composition of the Synthetic URINE SAlt MatriX

\begin{tabular}{ll}
\hline Component & Concentration \\
\hline $\mathrm{CaCl}_{2} \cdot 2 \mathrm{H}_{2} \mathrm{O}$ & $40 \mathrm{mg} \mathrm{L}^{-1}$ \\
$\mathrm{MgSO}_{4} \cdot 7 \mathrm{H}_{2} \mathrm{O}$ & $490 \mathrm{mg} \mathrm{L}^{-1}$ \\
$\mathrm{Na}_{2} \mathrm{SO}_{4} \cdot 10 \mathrm{H}_{2} \mathrm{O}$ & $3200 \mathrm{mg} \mathrm{L}^{-1}$ \\
$\mathrm{KHCO}_{3}$ & $2500 \mathrm{mg} \mathrm{L}^{-1}$ \\
$\mathrm{NaNO}_{3}$ & $150 \mathrm{mg} \mathrm{L}^{-1}$ \\
$\mathrm{FeSO}_{4} \cdot 7 \mathrm{H}_{2} \mathrm{O}$ & $1.4 \mathrm{mg} \mathrm{L}^{-1}$ \\
$\mathrm{KH}_{2} \mathrm{PO}_{4}$ & $1564 \mathrm{mg} \mathrm{L}^{-1}$ \\
$\mathrm{~K}_{2} \mathrm{HPO}_{4}$ & $2000 \mathrm{mg} \mathrm{L}^{-1}$ \\
$\mathrm{NaCl}$ & $5200 \mathrm{mg} \mathrm{L}^{-1}$ \\
\hline
\end{tabular}

vent oxygen limitation. The strains were harvested from the culture media, washed three times with phosphate-buffered saline, and inoculated in the synthetic urine salt matrix reported in Table 2 to an initial $\mathrm{OD}_{600}$ of 0.1 . To test the role of salinity, the medium was diluted to a final EC of 5, 10, and $30 \mathrm{mS} \mathrm{cm}$. Urea was added to a concentration of either 10,25 , or $50 \mathrm{mg}-\mathrm{N} \mathrm{L}^{-1}$ for each salinity tested. In all wells, $1 \mathrm{~g} \mathrm{~L}^{-1}$ of glucose was supplemented as a nonlimiting and easily degradable carbon source (Görke and Stülke, 2008), so that the cultures will only be limited by the salinity and the nitrogen. Ureolysis was evaluated as ammonia production for 5 days.

2.3.2. Effect of amino-acidic nitrogen. All the tests were assembled in a 3 -fold diluted synthetic urine salt matrix in $75 \mathrm{mM}$ phosphate buffer at $\mathrm{pH} 7$ (Table 2). Organic nitrogen was supplemented adding 20, 10,5 , or $0 \mathrm{ml} \mathrm{L}^{-1}$ of RPMI 1640 amino acid solution $50 \times($ Sigma), to a final amino acid-N concentration of 130 , resembling undiluted urine (Kim et al., 2013), 65, 33, and $0 \mathrm{mg} \mathrm{L}^{-1}$. Urea was added to a concentration of $100 \mathrm{mg}-\mathrm{N} \mathrm{L}^{-1}$. The ammonium produced by the metabolism of the amino acids was determined including controls without urea and subtracted from the total.

2.3.3. Nitrate production from synthetic urine with different synthetic communities. All the tests were assembled in a synthetic urine salt matrix diluted three times in $75 \mathrm{mM}$ phosphate buffer at pH 7 (Table 2) and supplemented with organic components to resemble real urine. More specifically, the carbon solution included Na-acetate $3.3 \mathrm{~g} \mathrm{~L}^{-1}$, RPMI amino acid solution $6.7 \mathrm{ml} \mathrm{L}^{-1}$, and L-glutamine $97 \mathrm{mg} \mathrm{L}^{-1}$. Urea was added to a concentration of $100 \mathrm{mg}-\mathrm{N}$ $\mathrm{L}^{-1}$. The activity was evaluated in triplicates in $10 \mathrm{~mL}$ test tubes containing a total volume of $3 \mathrm{~mL}$.

2.3.4. Clinostat: Hardware and assembly procedures. An overview of the hardware utilized to test the effect of clinorotation is presented in Fig. 1. The fluid processing apparatus (FPA) includes the glass barrels, rubber stoppers, and gas exchange insert (Bioserve Space Technologies, Boulder, $\mathrm{CO})$. All the components were coated with a silicone lubricant (Sigmacote, Sigma, St. Louis, MO) and autoclaved at $121^{\circ} \mathrm{C}$ for $20 \mathrm{~min}$. Two rubber stoppers and one gas exchange membrane were inserted in the glass barrels to obtain three compartments (Fig. 1a). The samples intended for clinorotation experiments were additionally provided with a cellulose membrane attached to the gas exchange membrane 

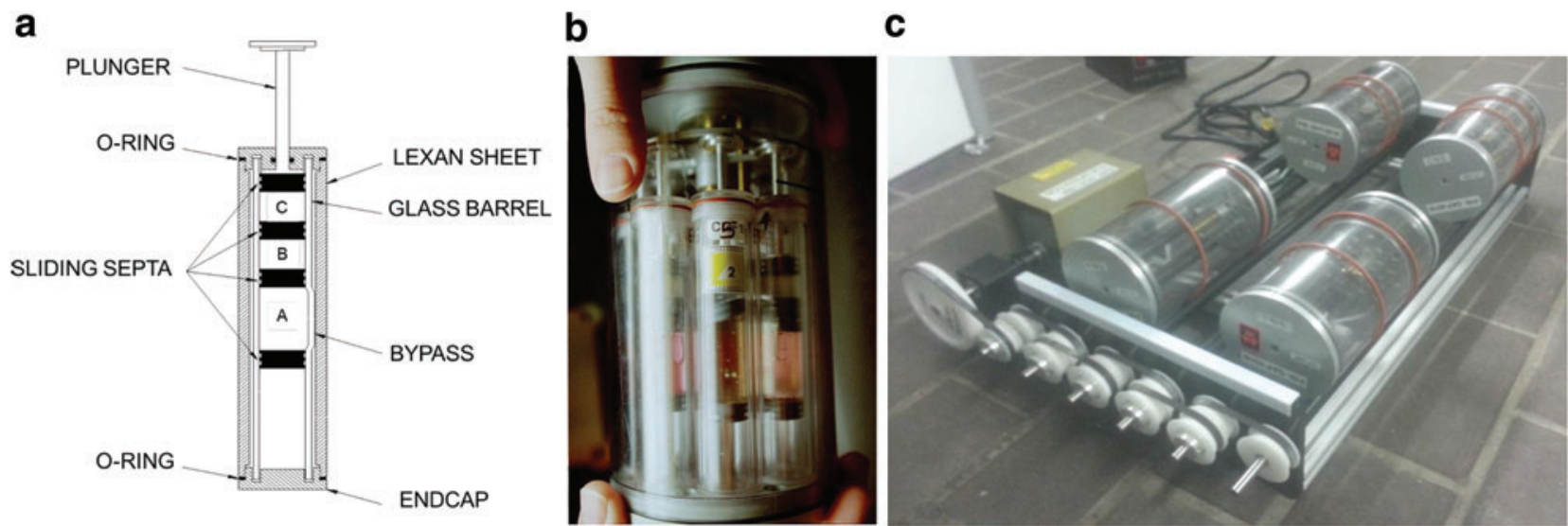

FIG. 1. (a) Schematic overview of the different compartments of a fluid processing apparatus. Exemplification of their positioning (b) in a group activation pack and (c) in the clinostat. Courtesy of BioServe Space Technologies at the University of Colorado. Color images are available online.

with a double-sided autoclavable tape. This step was performed to provide a surface for biofilm development and simulate biofilm experiment conditions. The three compartments were filled respectively with the microbial inoculum $(2.5 \mathrm{~mL})$, the substrate $(0.5 \mathrm{~mL})$, and the fixative $(2 \mathrm{~mL})$, following the manufacturer's instruction. Syringe needles were inserted into rubber stoppers during each step to release air pressure and prevent the formation of bubbles. Activation of the experiment was achieved by pushing a plunger until the inoculum and substrate compartment were mixed, while the experiment was terminated by pushing the plunger until the fixative was mixed.

Each FPA was loaded into a lexan sheet that contained a gas-permeable membrane at the bottom that was subsequently loaded into a specific plastic container, the group activation pack or GAP (Bioserve Space Technologies, Boulder, CO) (Fig. 1b). The GAPs for the test with the pure strains contained eight FPAs, while the ones for the test with the complete consortium held four, to reduce the competition for oxygen. The FPAs were activated simultaneously. Low-shear modeled microgravity was achieved by positioning the GAPs in a clinostat, a device that ensures constant rotation of the cultures perpendicularly to the gravitational pull, hence nullifying the effect of terrestrial gravity. In this work, a clinostat compatible with the FPA and GAP hardware was selected, namely the GAP-A540 HD Clinostat (Bioserve Space Technologies, Boulder, CO) (Fig. 1c).

In space, mass transport in a liquid medium is limited to Brownian motion given that gravity-driven forces and flowssedimentation, buoyancy, and convection-are not present, producing a quiescent extracellular environment where substrates lack and metabolic by-products abound (Klaus et al. 1997; Zea et al., 2016). While clinostats cannot remove the $1 g$ vector as they are operated on Earth, they serve to replicate some aspects of this altered extracellular environment by maintaining a cell suspended within a depletion zone. To do this, the clinostat angular speed is calculated as a function of cellular and medium properties, including but not limited to cell length and diameter (normalized into Stoke's diameter), mass, and density, as well as medium density and viscosity (Kessler 1992; Klaus, 2007; Zea et al., 2018).
The cellular length and diameter for the used bacterial models are as follows: Comamonas $0.6-0.8 \mu \mathrm{m}$ and 0.8 $2.0 \mu \mathrm{m}$ (Zhu et al., 2014), Nitrosomonas europaea 1.2$1.7 \mu \mathrm{m}$ and 1.0-1.2 $\mu \mathrm{m}$ (Meiklejohn, 1950), and Nitrobacter winogradskyi has $0.5 \mu \mathrm{m}$ and $1.0 \mu \mathrm{m}$, respectively (Zavarzin and Legunkova 1959). These were homogenized and simplified as a Stoke's diameter of $5.6 \times 10^{-5} \mathrm{~cm}$. Growth medium viscosity of $0.0102 \mathrm{~g} / \mathrm{cm} \cdot \mathrm{s}$ and density of $1.011 \mathrm{~g} / \mathrm{cm}^{3}$ (for reference, water's viscosity at $25^{\circ} \mathrm{C}$ and density are $0.0091 \mathrm{~g} / \mathrm{cm} \cdot \mathrm{s}$ and $1.000 \mathrm{~g} / \mathrm{cm}^{3}$, respectively), cell mass of $4.0 \times 10^{-13} \mathrm{~g}$, and an arbitrary quiescent zone diameter of $100 \mu \mathrm{m}$ were used as input parameters into BioServe's MathWork's MATLAB code based on the ode15s ordinary differential equation solver (Zea et al., 2018), to determine what would be the longest duration a cell would stay within the quiescent zone under these conditions as a function of angular speed. Three speeds were tested: 5,10 , and $30 \mathrm{rpm}$, resulting in 45,11 , and 1.25 days, respectively. We therefore chose $5 \mathrm{rpm}$ as the speed to rotate, given this experiment's incubation durations. While the code has its limitations, namely that it does not include Brownian motion, it serves as a first-order analysis to determine the best angular speed based on cellular and media parameters.

Cultures exposed to static $1 g$ conditions were included as controls to evaluate consequences of the gravity-induced settling of bacteria and particles. The static controls were placed horizontally on the same surface on which the clinostat is sitting, to ensure the vibrations from the motor are also experienced by the controls. Clinorotated and static controls were incubated at $28^{\circ} \mathrm{C}$ and activated simultaneously.

Pure cultures of C. testosteroni $\mathrm{I} 2$ as ureolytic heterotroph, N. europaea ATCC 19178 as ammonium oxidizer, and N. winogradskyi ATCC 25391 as nitrite oxidizer were utilized to validate the FPAs. The synthetic microbial community made of C. testosteroni, N. europaea, and $N$. winogradskyi, assembled as previously described, was utilized for the clinorotation experiment. Briefly, the strains were harvested from the culture media, washed three times with phosphate-buffered saline, and inoculated in a synthetic urine salt matrix, as previously described.

For $C$. testosteroni and the synthetic community, the substrate compartment was filled with a concentrated mix of 
the carbon compounds. Once the activation is performed, the final concentration obtained is the one present in a threetimes diluted synthetic urine (Na-acetate $3.3 \mathrm{~g} \mathrm{~L}^{-1}$, RPMI amino acid solution $6.7 \mathrm{ml} \mathrm{L}^{-1}$, L-glutamine $97 \mathrm{mg} \mathrm{L}^{-1}$, and urea $3.3 \mathrm{~g} \mathrm{~L}^{-1}$ ). For the test with $N$. europaea, the substrate consisted in a solution of 400 and $800 \mathrm{mg} \mathrm{L}^{-1} \mathrm{NH}_{4}^{+}-\mathrm{N}$, while for the test with only $N$. winogradskyi, 400 and $800 \mathrm{mg} \mathrm{L}^{-1} \mathrm{NO}_{2}-\mathrm{N}$ were used. The fixative utilized was a solution of $9 \%$ paraformaldehyde.

\section{Results}

\subsection{Ureolysis is inhibited at an EC of $30 \mathrm{mS} \mathrm{cm}^{-1}$}

The ureolytic activity tests performed with heterotrophic pure cultures showed no significant difference among the conditions of $5 \mathrm{mS} \mathrm{cm}^{-1}$ and $10 \mathrm{mS} \mathrm{cm}^{-1}$ (Fig. 2). However, ammonia production could not be detected in any culture exposed to $30 \mathrm{mS} \mathrm{cm} \mathrm{cm}^{-1}$.

\subsection{Acidovorax delafieldii, Cupriavidus necator and Delftia acidovorans require minimal urea concentrations to display ureolytic activity}

Ammonia production was observed for all heterotrophs exposed to the highest concentration of urea $(50 \mathrm{mg}$ urea-N $\mathrm{L}^{-1}$ ), with $A$. delafieldii displaying the highest rates at both 5 and $10 \mathrm{mS} \mathrm{cm}^{-1}\left(40.0 \pm 0.2\right.$ and $32.4 \pm 3.7 \mathrm{mg} \mathrm{NH}_{4}^{+}-\mathrm{N} \mathrm{L}^{-1} \mathrm{~d}^{-1}$, respectively) (Fig. 2). Only C. testosteroni, P. fluorescens, and $V$. campbellii displayed ammonia production at lower urea concentrations.

\subsection{Amino-acidic nitrogen mildly inhibits ureolysis}

The presence of amino acid-N affected urea hydrolysis in all the strains tested (Fig. 3).

Vibrio campbellii and Acidovorax delafieldii are not affected by the presence of amino acids. Cupriavidus necator, Delftia acidovorans, and Comamonas testosteroni show amino acidic nitrogen inhibition at 65 and $130 \mathrm{mg}$ amino acid $\mathrm{N} \mathrm{L}^{-1}$ ( $p$ value $\left.<0.05\right)$. Pseudomonas fluorescens shows a 3-fold decrease in urea hydrolysis rates at the highest amino acid concentration tested, $130 \mathrm{mg}$ amino acid $\mathrm{N}$ $\mathrm{L}^{-1}(p$ value $<0.05)$. Its rates at lower concentrations are comparable.

\subsection{Urea hydrolysis and nitrification is confirmed for all microbial communities, except for those including Cupriavidus necator or Vibrio campbellii}

Ammonium was available for oxidation in all cultures (Fig. 4a). In most communities, its concentration increased within the first 5 days due to the hydrolysis of urea, reaching its maximum concentration between days 5 and 10. Communities with $A$. delafieldii displayed the fastest ureolytic activity. Communities with $P$. fluorescens showed a delayed urease activity, which began only after day 5 and was completed at day 25 .

The total nitrogen flux channeled toward nitrification can be evaluated as the sum of nitrite and nitrate (Fig. 4b), which also provides an indication on the ammonia oxidation activity of $N$. europaea. Nitrification occurred in all the synthetic microbial communities, except for the ones with $C$. necator and $V$. campbellii. The presence in the synthetic communities of $P$. fluorescens or $C$. testosteroni is beneficial for ammonia oxidation more than the presence of $A$. delafieldii and $D$. acidovorans, as the rates were 2 times higher. Nitrite accumulation was observed in all the synthetic microbial communities (Fig. 4c), except for the controls without heterotroph. At the end of the batch test (day 24), the synthetic community with $P$. fluorescens showed a nitrite accumulation significantly higher than $D$. acidovorans ( $p$ value $<0.05$ ) but comparable to the one of $C$. testosteroni. The production of nitrate, the preferred fertilizing nitrogen species in MELiSSA, is the slowest conversion, and the final concentration of nitrate was the lowest among all metabolites monitored (Fig. 4d).

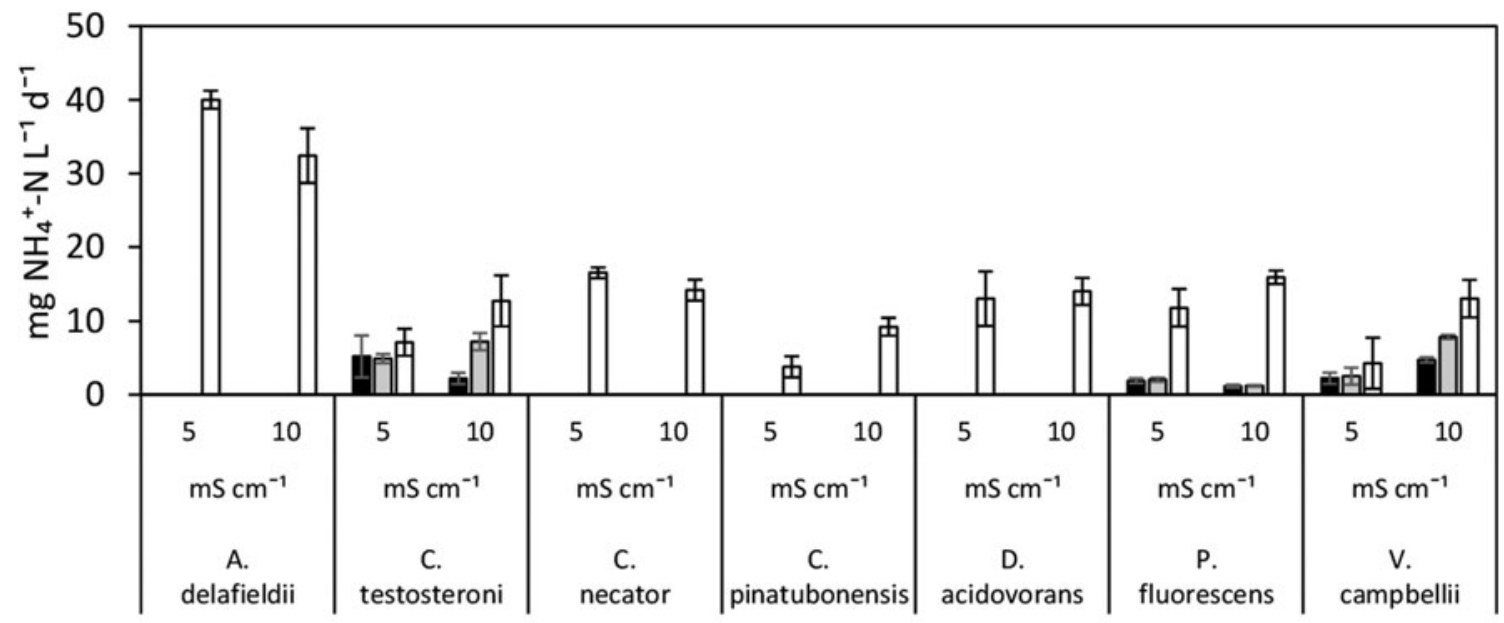

$10 \mathrm{mg}$ urea- $\mathrm{N} \mathrm{L}^{-1}$ $25 \mathrm{mg}$ urea- $\mathrm{N} \mathrm{L}^{-1}$ $\square 50 \mathrm{mg}$ urea-N L ${ }^{-1}$

FIG. 2. Ammonia production rate $\left(\mathrm{mg} \mathrm{N} \mathrm{L}^{-1} \mathrm{~d}^{-1}\right)$ by different heterotrophs in the presence of 10,25 , and $50 \mathrm{mg}$ urea-N $\mathrm{L}^{-1}$ and at two different salinities $\left(5\right.$ and $\left.10 \mathrm{mS} \mathrm{cm}^{-1}\right)$. Error bars represent the standard deviation of three replicate cultures. 


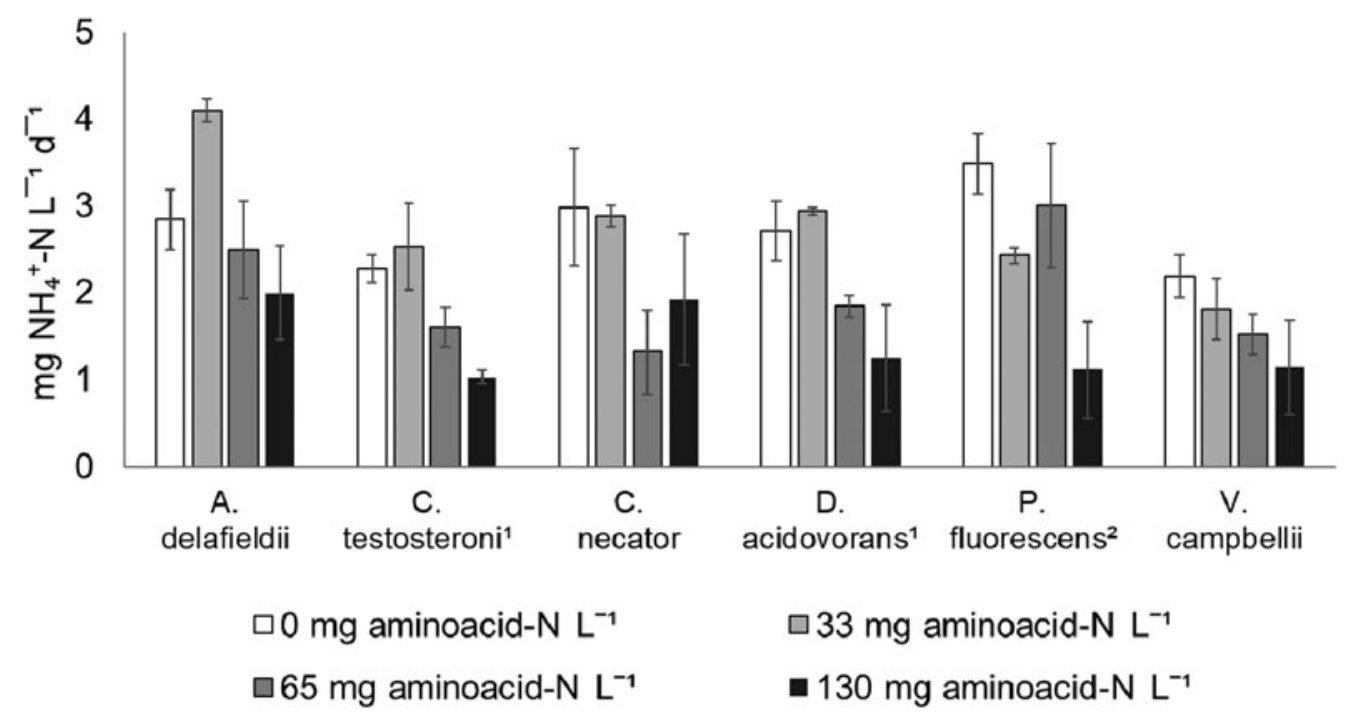

FIG. 3. Overview of the average $(n=3)$ urea hydrolysis (evaluated as ammonia production rates), in the presence of 0,33 , 65 , and $130 \mathrm{mg}$ amino acid-N L $\mathrm{L}^{-1}$. The mark $\left(^{1}\right)$ indicates cultures whose ammonia production is significantly different in the presence of 65 and $130 \mathrm{mg}$ amino acid- $\mathrm{N} \mathrm{L}^{-1}$. The mark $\left({ }^{2}\right)$ indicates cultures whose ammonia production is significantly different in the presence of $130 \mathrm{mg}$ amino acid-N L ${ }^{-1}$.
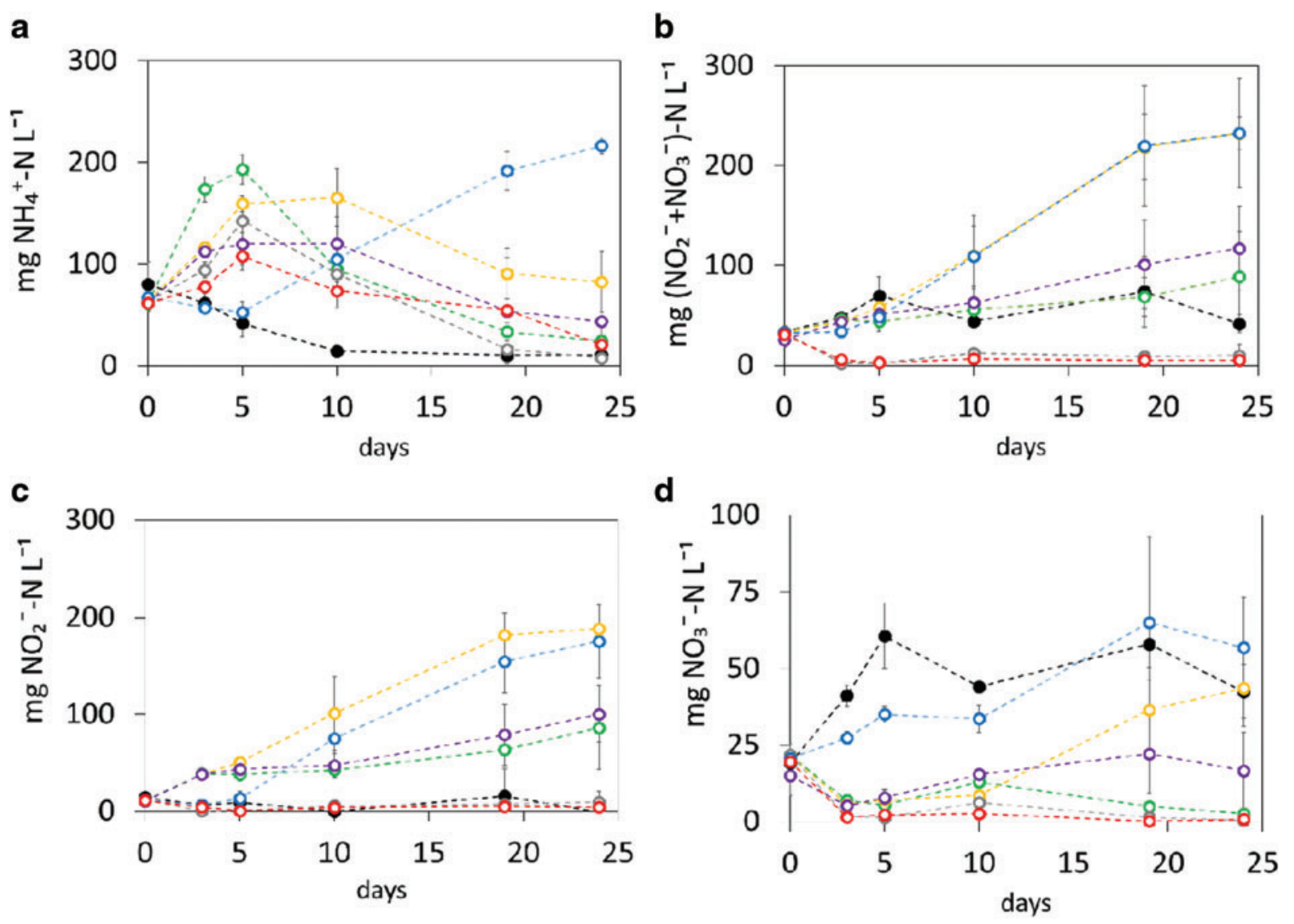

$$
\begin{aligned}
& \text { - Nitrosomonas + Nitrobacter (Nitr) } \\
& - \text { Nitr + C. testosteroni } \\
& \text { - Nitr + D. acidovorans } \\
& \text { - - Nitr + V. campbellii }
\end{aligned}
$$

$$
\begin{aligned}
& \text { - Nitr + A. delafieldii } \\
& \text { o-Nitr + C. necator } \\
& \text { o-Nitr + P. fluorescens }
\end{aligned}
$$

FIG. 4. Ammonia (a), nitrite + nitrate (b), nitrite (c), and nitrate (d) levels as a function of time for synthetic communities composed of $N$. europaea, $N$. winogradskyi, and the different heterotrophs in the presence of $100 \mathrm{mg}^{-\mathrm{N} \mathrm{L}} \mathrm{L}^{-1}$. Nitrogen was added as ammonia for the community composed of $N$. europaea and $N$. winogradskyi only, and as urea for the other communities. Error bars represent the standard deviation among triplicates. Color images are available online. 
a

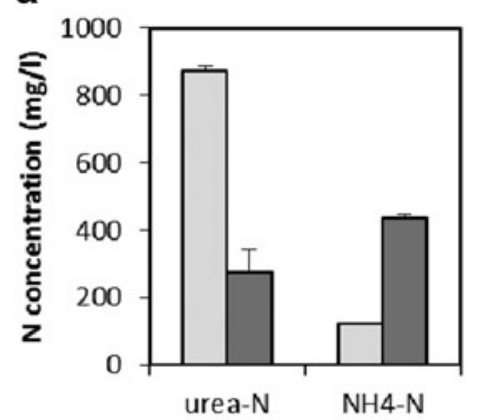

b

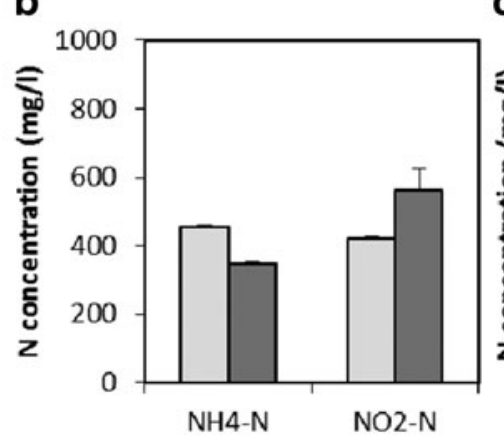

C

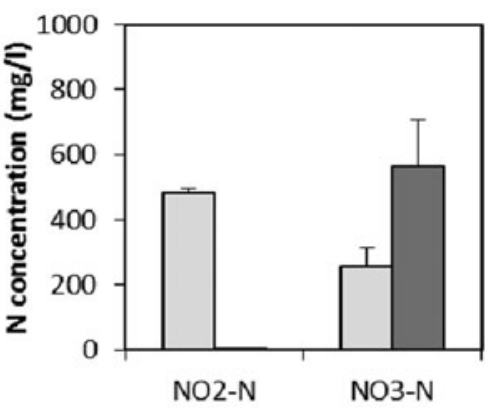

$\square$ start $\square$ end

FIG. 5. (a) Urea-N and $\mathrm{NH}_{4}{ }^{+}-\mathrm{N}$ concentrations in the FPA tubes at the start and end (2 weeks) of the experiment with $C$. testosteroni I2. (b) $\mathrm{NH}_{4}{ }^{+}-\mathrm{N}$ and $\mathrm{NO}_{2}{ }^{-}-\mathrm{N}$ concentrations in the FPA tubes inoculated with $N$. europaea at the start and end (3 weeks) of the experiment. (c) $\mathrm{NO}_{2}^{-}{ }^{-} \mathrm{N}$ and $\mathrm{NO}_{3}{ }^{-} \mathrm{N}$ concentrations in the FPA tubes inoculated with $N$. winogradskyi at the start and end ( 2 weeks) of the experiment. Error bars represent the standard deviation of three replicate cultures.

The microbial community with $P$. fluorescens produced nitrate in the first 3 days and up to $65 \pm 28 \mathrm{mg} \mathrm{NO}_{3}^{-}-\mathrm{N} \mathrm{L}^{-1}$ in the first 20 days. All other heterotrophic communities show a decrease in the $\mathrm{NO}_{3}{ }^{-}$levels present in the original medium in the first 5 days. The production of nitrate resumed in the communities with $C$. testosteroni and D. acidovorans. Acidovorax delafieldii shows a limited production of $\mathrm{NO}_{3}{ }^{-}$, reaching a maximum concentration of $13 \pm 2 \mathrm{mg} \mathrm{NO}_{3}^{-}-\mathrm{N} \mathrm{L}^{-1}$.

\subsection{All monocultures are active in the clinostat component. Microbial activity of the synthetic community is not affected by low-shear modeled microgravity exposure}

To validate the different components of the clinostat hardware, the activity of the different pure cultures was tested in the FPA tubes and GAP containers without clinorotation. Comamonas testosteroni hydrolyzed almost $70 \%$ of the urea, resulting in a net ammonia production of $315 \mathrm{mg}$ $\mathrm{NH}_{4}{ }^{+}-\mathrm{N} \mathrm{L} \mathrm{L}^{-1}$ (Fig. 5a). Nitrosomonas europaea oxidized $25 \%$ of the ammonium-N within the timeframe of the experiment ( 3 weeks), and this was reflected in an increase in

a

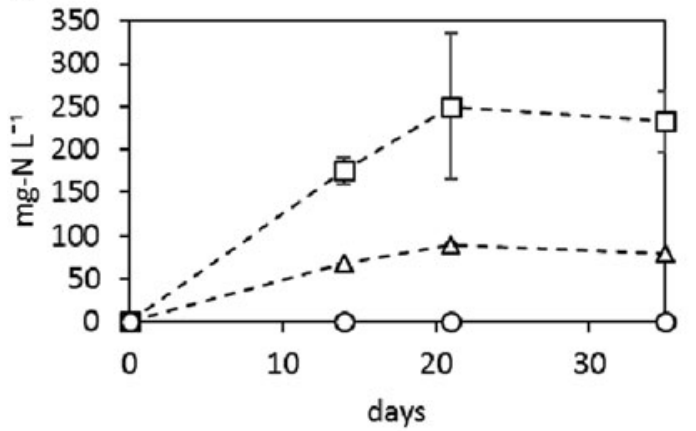

the nitrite concentration of $141 \mathrm{mg} \mathrm{NO}_{2}^{-}-\mathrm{N} \mathrm{L}^{-1}$ (Fig. 5b). Nitrobacter winogradskyi completely oxidized the nitrite present in the medium within 2 weeks, and this was reflected in an increase in the nitrate concentration (Fig. 5c).

The synthetic community of C. testosteroni, N. europaea, and $N$. winogradskyi exposed to low-shear modeled microgravity through clinorotation showed similar behavior as the static control (Fig. 6). Ammonia production could be observed in the first 20 days, indicating the ureolytic activity of C. testosteroni. Although the samples exposed to clinorotation showed higher ammonia levels than the static controls, the large standard deviation makes this observation inconclusive. Nitrite production, which is associated with the activity of $N$. europaea, was observed in the same time frame. After 20 days, its accumulation stopped, and the concentration remained constant. No nitrate production was observed in the time frame of the experiment, indicating that N. winogradskyi did not perform nitrite oxidation (Fig. 6).

\section{Discussion}

The results obtained by testing different heterotrophs highlight the possibility of diluting the urine matrix to cope

b

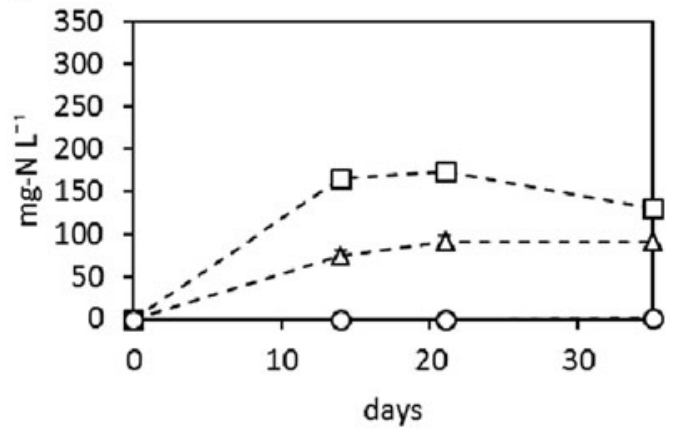

FIG. 6. Overview of the ammonia, nitrite, and nitrate concentrations over time in synthetic microbial communities exposed to clinorotation (a) and static controls (b). Error bars represent the standard deviation among triplicates. 
with the inhibition observed at higher salinity. In fact, none of the strains showed urea hydrolysis at the highest EC (30 $\mathrm{mS} \mathrm{cm} \mathrm{cm}^{-1}$ ), and all required a salinity of 5 or $10 \mathrm{mS} \mathrm{cm}$ to function. Although most of the edible plants considered for regenerative life-support systems require low $\mathrm{EC}$ in the hydroponic nutrient solution (Tibbits and Alford, 1982), the opportunity of incorporating halophytic edible plant cultivation such as the salt-accumulating Salicornia europaea and the salt-secreting Limonium gmelinii on urine has been proposed (Balnokin et al., 2010; Tikhomirova et al., 2011). This represents an elegant approach to recycle $\mathrm{NaCl}$ and save water, although the limited nutritional value and use in culinary preparations could hamper their use. Both plants could be successfully grown on nutrient solutions containing mineralized human urine. This however resulted in lower biomass yield than that of controls (Tikhomirova et al., 2011). The application of nitrified urine was not evaluated. In a scenario in which urine nitrification will be required at high EC, for the cultivation of halophytes, salt adaptation of the microbial inoculum is a promising option (Coppens et al., 2016). Alternatively, although still requiring optimization, selective ion removal to ensure the optimal salinity can be obtained through electrodialysis, salt precipitation, or electrochemical technology (De Paepe et al., 2018). The optimal dilution for efficient urea hydrolysis should be evaluated taking into account the fact that some strains show ureolytic activity only at specific urea concentrations. The response of ureolytic bacteria to urea and organic nitrogen is commonly strain dependent. While some reports indicate that urease synthesis is repressed in the presence of ammonia, urea, or nitrogen-rich compounds, urea serves as ureolysis inducer in other bacteria. Some other strains possess a constitutive expression of urease (Mobley and Hausinger, 1989). Of the strains tested, only C. testosteroni, $V$. campbellii, and $P$. fluorescens showed ammonia production at low urea concentration. The presence of aminoacidic nitrogen proved to slow the rates of all the strains tested, at concentrations comparable to the ones in undiluted urine (Kim et al., 2013). Even if the difference amounts to only a few mg-N L ${ }^{-1} \mathrm{~d}^{-1}$, the dilution of urine can minimize this unwanted effect. Recalcitrant organic molecules, however, can accumulate with time, possibly reaching an inhibitory threshold, requiring long-term and in specific analysis for their identification.

Overall, the use of bacteria that present different responses to the environmental concentration of urea and organic nitrogen will ensure the active expression of the ureolytic enzymes, regardless of the matrix dilution.

The analysis of synthetic communities identified positive microbial interaction and confirmed for the first time the production of nitrate in a simplified synthetic urine matrix. Generally, the presence of a third heterotrophic strain negatively affects the process, causing a nitrite buildup. This can be potentially harmful for $N$. europaea, which is inhibited by high levels of nitrite (and free nitrous acid). Additionally, it possess detoxifying mechanisms that can lead to nitrogen losses and the production of nitrogen gas $\left(\mathrm{N}_{2}\right)$, NO, and $\mathrm{N}_{2} \mathrm{O}$ (Stein, 2011). This nitrite accumulation can be caused by a positive effect of the heterotroph on $N$. europaea, increasing the ammonia oxidation and rendering nitrite oxidation the bottleneck of the process. Indeed, the presence of heterotrophic strains has been described as beneficial for an estuarine AOB, Nitrosomonas sp. The ammonia oxidation increased by as much as $150 \%$ and $50 \%$ when grown in the presence of Nocardia atlantica and Pseudomonas sp. isolated from the same environment. The growth of the heterotrophs was one order of magnitude higher in the presence of the ammonia oxidizer (Jones and Hood, 1980). The positive and mutual interaction has been studied under selective conditions and linked with the utilization of heterotrophic siderophores by $N$. europaea, which produces small organic compounds (such as pyruvate) that can be metabolized by the heterotroph (Keluskar et al., 2013). The nitrite accumulation could also be generated by a negative interaction between heterotrophs and $N$. winogradskyi. Few reports address this type of interaction, and tests are mainly performed with microbial cultures with undefined composition. Yet one study observed a positive effect of a Pseudomonas sp. on N. winogradskyi, as shown by a higher activity, shorter latency, and increase in the growth rate (Blanc et al., 1986). The introduction of a heterotrophic strain, with a fast aerobic metabolism, can also have a third effect: inducing competition between $N$. europaea and $N$. winogradskyi for dissolved oxygen. It has been reported that the decrease of dissolved oxygen level is limiting for the oxidation of nitrite more than for ammonium, causing nitrite accumulation. Indeed, Km (half saturation constant) values for AOB are commonly between 1 and $15 \mu \mathrm{M} \mathrm{O}_{2}$ and between 22 and $166 \mu \mathrm{M} \mathrm{O}_{2}$ for the NOB. Nitrosomonas europaea is particularly competitive and possesses the lowest $\mathrm{Km}$ values (Laanbroek and Gerards, 1993).

This disparity in $\mathrm{Km}$ is also probably responsible for the results obtained in the clinorotation experiments. While the validation of the hardware was successful for the strain tested individually, no nitrite oxidation could be observed in the clinorotation experiments with the synthetic community for complete urea hydrolysis and subsequent nitrification. To enhance $N$. winogradskyi activity during in-flight tests, better aeration of the FPA and GAP should be ensured, possibly diluting the substrate.

The heterotroph generally had a negative effect on the nitrification process, and particularly $C$. necator and $V$. campbellii proved to be detrimental. Since ammonia was available, the suppression of nitrification could be caused by inhibiting compounds produced by the heterotroph, although specific tests are required to confirm the cause of this inhibition. However, nitrate could be successfully accumulated in the communities with $P$. fluorescens, D. acidovorans, and C. testosteroni.

\section{Conclusion}

This study presents the implementation of a functioning nitrifying consortium ( $N$. europaea and $N$. winogradskyi) with ureolytic and nonpathogenic heterotrophs, for potential use in urine nitrification systems for deep space exploration. The strains tested were Acidovorax delafieldii DSMZ 64, Comamonas testosteroni I2, Cupriavidus necator DSMZ 13513, Delftia acidovorans DSMZ 14801, Pseudomonas fluorescens DSMZ 50090, and Vibrio campbellii LMG 22895. The results obtained demonstrate the need to treat a diluted urine matrix when utilizing the tested microorganisms, although salt adaptation could be utilized as a strategy 
to reduce the dilution required. Among the strains, the communities with $P$. fluorescens, D. acidovorans, and C. testosteroni proved to be valid candidates for application in regenerative life-support systems. While oxygen limitation is most likely the limiting step on the functionality of the complete bioprocess in the clinostat hardware, microgravity had no impact, which is encouraging for the application of this process in space.

Successful nitrate production from urea was demonstrated utilizing synthetic microbial communities with different composition, supporting full-scale urine nitrification in regenerative life-support systems and human space exploration.

\section{Acknowledgments}

This article has been made possible through the authors' involvement in the MELiSSA project, ESA's life-support system research program http://www.esa.int/Our_Activities/ Space_Engineering_Technology/Melissa. We kindly acknowledge the financial support of the Belgian Science Policy (BELSPO).

\section{References}

Andrews, M., Raven, J., and Lea, P. (2013) Do plants need nitrate? The mechanisms by which nitrogen form affects plants. Ann Appl Biol 163:174-199.

Balnokin, Y., Myasoedov, N., Popova, L., Tikhomirov, A., Ushakova, S., Lasseur, C., and Gros, J.B. (2010) Use of halophytic plants for recycling $\mathrm{NaCl}$ in human liquid waste in a bioregenerative life support system. Adv Space Res 46:768774.

Benoit, M.R. and Klaus, D.M. (2007) Microgravity, bacteria, and the influence of motility. Adv Space Res 39:1225-1232.

Blanc, J., Audic, J., and Faup, G. (1986) Enhancement of $\mathrm{Ni}$ trobacter activity by heterotrophic bacteria. Water Res 20: 1375-1381.

Boon, N., Goris, J., De Vos, P., Verstraete, W., and Top, E.M. (2000) Bioaugmentation of activated sludge by an indigenous 3-chloroaniline-degrading Comamonas testosteroni strain, I2gfp. Appl Environ Microbiol 66:2906-2913.

Bouatra, S., Aziat, F., Mandal, R., Guo, A.C., Wilson, M.R., Knox, C., Bjorndahl, T.C., Krishnamurthy, R., Saleem, F., and Liu, P. (2013) The human urine metabolome. PloS One 8, doi:10.1371/journal.pone.0073076.

Brenner, K., You, L., and Arnold, F.H. (2008) Engineering microbial consortia: a new frontier in synthetic biology. Trends Biotechnol 26:483-489.

Bucur, B., Catala Icardo, M., and Martinez Calatayud, J. (2006) Spectrophotometric determination of ammonium by an rFIA assembly. Rev Roum Chim 51:101.

Cataldo, D., Maroon, M., Schrader, L., and Youngs, V. (1975) Rapid colorimetric determination of nitrate in plant tissue by nitration of salicylic acid. Commun Soil Sci Plant Anal 6: 71-80.

Clauwaert, P., Muys, M., Alloul, A., De Paepe, J., Luther, A., Sun, X., Ilgrande, C., Christiaens, M.E., Hu, X., Zhang, D., Lindeboom, R.E.F, Sas, B., Rabaey, K., Boon, N., Ronss, F., Geelen, D., and Vlaeminck, S.E. (2017) Nitrogen cycling in bioregenerative life support systems: challenges for waste refinery and food production processes. Progress in Aerospace Sciences 91:87-98.

Coppens, J., Lindeboom, R., Muys, M., Coessens, W., Alloul, A., Meerbergen, K., Lievens, B., Clauwaert, P., Boon, N., and
Vlaeminck, S.E. (2016) Nitrification and microalgae cultivation for two-stage biological nutrient valorization from source separated urine. Bioresour Technol 211:41-50.

Cruvellier, N., Poughon, L., Creuly, C., Dussap, C.-G., and Lasseur, C. (2016) Growth modelling of Nitrosomonas europaea ATCC ${ }^{\circledR} 19718$ and Nitrobacter winogradskyi ATCC $^{\circledR}$ 25391: a new online indicator of the partial nitrification. Bioresour Technol 220:369-377.

Daims, H., Lebedeva, E.V., Pjevac, P., Han, P., Herbold, C., Albertsen, M., Jehmlich, N., Palatinszky, M., Vierheilig, J., and Bulaev, A. (2015) Complete nitrification by Nitrospira bacteria. Nature 528:504-509.

Defoirdt, T., Vlaeminck, S.E., Sun, X., Boon, N., and Clauwaert, P. (2017) Ureolytic activity and its regulation in Vibrio campbellii and Vibrio harveyi in relation to nitrogen recovery from human urine. Environ Sci Technol 51:13335-13343.

De Paepe, J., Lindeboom, R.E.F., Vanoppen, M., De Paepe, K., Demey, D., Coessens, W., Lamaze, B., Verliefde, A.R.D., Clauwaert, P., and Vlaeminck, S.E. (2018) Refinery and concentration of nutrients from urine with electrodialysis enabled by upstream precipitation and nitrification. Water Res 144:76-86.

EPA. (2016) IRIS Toxicological Review of Ammonia Noncancer Inhalation, Final Report, U.S. Environmental Protection Agency, Washington, DC.

Görke, B. and Stülke, J. (2008) Carbon catabolite repression in bacteria: many ways to make the most out of nutrients. Nat Rev Microbiol 6:613-624.

Hendrickx, L. and Mergeay, M. (2007) From the deep sea to the stars: human life support through minimal communities. Curr Opin Microbiol 10:231-237.

Hendrickx, L., De Wever, H., Hermans, V., Mastroleo, F., Morin, N., Wilmotte, A., Janssen, P., and Mergeay, M. (2006) Microbial ecology of the closed artificial ecosystem MELiSSA (Micro-Ecological Life Support System Alternative): reinventing and compartmentalizing the Earth's food and oxygen regeneration system for long-haul space exploration missions. Res Microbiol 157:77-86.

Jetten, M.S. (2008) The microbial nitrogen cycle. Environ Microbiol 10:2903-2909.

Jones, R.D. and Hood, M.A. (1980) Interaction between an ammonium-oxidizer, Nitrosomonas sp., and two heterotrophic bacteria, Nocardia atlantica and Pseudomonas sp.: a note. Microb Ecol 6:271-275.

Keluskar, R., Nerurkar, A., and Desai, A. (2013) Mutualism between autotrophic ammonia-oxidizing bacteria (AOB) and heterotrophs present in an ammonia-oxidizing colony. Arch Microbiol 195:737-747.

Kessler, J.O. (1992) The internal dynamics of slowly rotating biological systems. Gravitational and Space Research 5:1121.

Kim, W., Tengra, F.K., Young, Z., Shong, J., Marchand, N., Chan, H.K., Pangule, R.C., Parra, M., Dordick, J.S., and Plawsky, J.L. (2013) Spaceflight promotes biofilm formation by Pseudomonas aeruginosa. PloS One 8, doi:10.1371/journal.pone.0062437.

Klaus, D., Simske, S., Todd, P., and Stodieck, L. (1997) Investigation of space flight effects on Escherichia coli and a proposed model of underlying physical mechanism. Microbiology 143:449-455.

Klaus, D.M. (2007) Clinostats and bioreactors. Gravitational and Space Research 14:55-64.

Laanbroek, H.J. and Gerards, S. (1993) Competition for limiting amounts of oxygen between Nitrosomonas europaea and 
Nitrobacter winogradskyi grown in mixed continuous cultures. Arch Microbiol 159:453-459.

Lindeboom, R.E.F., Ilgrande, C., Carvajal-Arroyo, J.M., Coninx, I., Van Hoey, O., Roume, H., Morozova, J., Udert, K.M., Sas, B., Paille, C., Lasseur, C., Ilyin, V., Clauwaert, P., Leys, N., and Vlaeminck, S.E. (2018) Nitrogen cycle microorganisms can be reactivated after space exposure. Sci Rep 8, doi: 10.1038/s41598-018-32055-4.

Meiklejohn, J. (1950) The isolation of Nitrosomonas europaea in pure culture. Microbiology 4:185-191.

Mobley, H. and Hausinger, R. (1989) Microbial ureases: significance, regulation, and molecular characterization. Microbiol Rev 53:85-108.

Mobley, H., Island, M.D., and Hausinger, R.P. (1995) Molecular biology of microbial ureases. Microbiol Rev 59:451480.

Montgomery, H.A.C. and Dymock, J.F. (1961) The determination of nitrite in water. Analyst 86:414-416.

Montràs, A., Pycke, B., Boon, N., Gòdia, F., Mergeay, M., Hendrickx, L., and Pérez, J. (2008) Distribution of $\mathrm{Ni}$ trosomonas europaea and Nitrobacter winogradskyi in an autotrophic nitrifying biofilm reactor as depicted by molecular analyses and mathematical modelling. Water Res 42:1700-1714.

Muys, M., Coppens, J., Boon, N., and Vlaeminck, S.E. (2018) Photosynthetic oxygenation for urine nitrification. Water Sci Technol 78:183-194.

Nagaraj, N. and Mann, M. (2011) Quantitative analysis of the intra-and inter-individual variability of the normal urinary proteome. J Proteome Res 10:637-645.

Nickerson, C.A., Ott, C.M., Wilson, J.W., Ramamurthy, R., and Pierson, D.L. (2004) Microbial responses to microgravity and other low-shear environments. Microbiol Mol Biol Rev 68: 345-361.

Pérez, J., Montesinos, J., Albiol, J., and Godia, F. (2004) Nitrification by immobilized cells in a micro-ecological life support system using packed-bed bioreactors: an engineering study. J Chem Technol Biotechnol 79:742-754.

Siener, R. and Hesse A. (2002) The effect of different diets on urine composition and the risk of calcium oxalate crystallisation in healthy subjects. Eur Urol 42:289-296.

Stein, L.Y. (2011) Heterotrophic nitrification and nitrifier denitrification. In Nitrification, American Society of Microbiology, Washington, DC, pp 95-114.

Tibbits, T. and Alford, D.K., editors. (1982) Controlled Ecological Life Support System: Use of Higher Plants, NASACP-2231, NASA, Washington, DC.

Tikhomirova, N., Ushakova, S., Kudenko, Y.A., Gribovskaya, I., Shklavtsova, E., Balnokin, Y.V., Popova, L., Myasoedov, N., Gros, J.-B., and Lasseur, C. (2011) Potential of saltaccumulating and salt-secreting halophytic plants for re- cycling sodium chloride in human urine in bioregenerative life support systems. Adv Space Res 48:378-382.

van Kessel, M.A., Speth, D.R., Albertsen, M., Nielsen, P.H., den Camp, H.J.O., Kartal, B., Jetten, M.S., and Lücker, S. (2015) Complete nitrification by a single microorganism. Nature 528:555-559.

Witte, C.-P. (2011) Urea metabolism in plants. Plant Sci 180: 431-438.

Yatzidis, H., Garidi, M., Vassilikos, C., Mayopoulou, D., and Akilas, A. (1964) An improved method for the simple and accurate colorimetric determination of urea with Ehrlich's reagent. Journal of Clinical Pathology 17:163-164.

Zavarzin, G. and Legunkova, R. (1959). The morphology of Nitrobacter winogradskyi. Microbiology 21:186-190.

Zea, L., Prasad, N., Levy, S.E., Stodieck, L., Jones, A., Shrestha, S., Stodieck, L., and Klaus, D. (2016) A molecular genetic basis explaining altered bacterial behavior in space. PloS One 11, doi:10.1371/journal.pone.0164359.

Zea, L., Estante, F., Rosengren, A., Stodieck, L., and Klaus, L. (2018) Determining an appropriate clinostat rotational speed. In 34th Annual Meeting of the American Society for Gravitational and Space Research (ASGSR), ASGSR, Springfield, VA.

Zhu, D., Xie, C., Huang, Y., Sun, J., and Zhang, W. (2014) Description of Comamonas serinivorans sp. nov., isolated from wheat straw compost. Int J Syst Evol Microbiol 64:4141-4146.

Address correspondence to: Nico Boon Center for Microbial Ecology and Technology Ghent University Coupure Links 653 9000 Gent Belgium

E-mail: nico.boon@ugent.be

Submitted 22 October 2018 Accepted 1 August 2019

Abbreviations Used
$\mathrm{AOB}=$ ammonia-oxidizing bacteria
$\mathrm{EC}=$ electrical conductivity
$\mathrm{FPA}=$ fluid processing apparatus
$\mathrm{GAP}=$ group activation pack
MELiSSA $=$ Micro-Ecological Life Support System
Alternative
$\mathrm{NOB}=$ nitrite-oxidizing bacteria

